\title{
Masticatory Performance In Subjects With Class II Treated With Myofunctional Appliances: A Review
}

\author{
Vesna Jankulovka ${ }^{1}$ Evdokija Jankulovska ${ }^{2}$, Vera Petrova ${ }^{3}$, \\ Gabriela Kjurchieva-Chuchkova ${ }^{4}$, Lidija Kanurkova ${ }^{5}$, Elena Petrova ${ }^{6}$, \\ Vaska Antevska ${ }^{7}$ \\ ${ }^{1}$ Private Dental Practice “JANKULOVSKI”, Skopje, Macedonia \\ ${ }^{2}$ Department Of Prosthodontics, Faculty Of Dentistry, Skopje, Macedonia \\ ${ }^{3}$ Department Of Neurology, Faculty Of Medicine, Skopje, Macedonia \\ ${ }^{4}$ Department Of Orthodontics, Faculty Of Dentistry, Skopje, Macedonia \\ ${ }^{5}$ Department Of Orthodontics, Faculty Of Dentistry, Skopje, Macedonia \\ ${ }^{6}$ Department Of Orthodontics, University Dental Clinical Center "Sveti Pantelejmon", Skopje, Macedonia \\ ${ }^{7}$ Department Of Medical And Experimental Physiology, Faculty Of Medicine, Skopje, Macedonia
}

\begin{abstract}
Aim: To present the results obtained from several different studies of the muscle activity of masticatory and perioral muscles in subjects with class II, as well as the effects of myofunctional appliances on the muscle activity during the treatment of Class II, harmful oral habits and soft tissue dysfunctions.

Materials and Methods: This review included 10 studies searched online in the PubMed database, which applied functional methods for masticatory evaluation. The search was carried out under the key words "mastication" "masticatory efficiency", "Class II," "myofunctional therapy", "electromyography", "masticatory muscles."

Results and Discussion: The results show that most of the authors in their researches applied the electromyography as a neurophysiological, graphical method for objective assessment of mastication and determination of the characteristics of EMG muscle activity of masticatory and perioral muscles.

Conclusions: According to the electromyographic characteristic of masseteric muscle activity, there are differences in the masticatory activity in subjects with Class I and Class II, as well as between Class II/I and Class II/2. The myofunctional appliances have a positive impact on the masticatory function.
\end{abstract}

Keywords: class II, mastication, masticatory efficiency, myofunctional appliances

\section{Introduction}

Since the beginning of the XX-th century, researchers realized the need of finding a simple and exact method to determine the masticatory function and efficiency, whose initial aim was to evaluate the success of dental restorative procedures. The first attempts to track down the exact methods of determining masticatory efficiency were made in the 3rd decade of the last century. The first step was undertaken by Cristiansen in 1924, and since then the effect of mastication became target of many studies in the global dental literature.

The processing of food during the masticatory act is a complex process that involves biomechanical, enzymatic and bacteriological processes. However, despite the complex nature of masticatory act, attention has been directed mostly to the mechanical processing of food. So far, different methods of testing and evaluation of the mechanical processing of food were described through literature, classified into 3 groups: static methods, static - functional methods and functional methods.

An objective assessment of the functional state of masticatory system is performed based on two basic principles: by determining the end result of mechanical grinding of food and through analysis of recorded movements of the lower jaw and masticatory muscles during mastication (graphical methods).

Electromyography is a graphic method for determining the masticatory efficiency. It is a neurophysiological graphical method for registration of muscle bioelectric potentials. Its application in orthodontic research area is enormous. By analyzing the electromyogram, we can obtain a lot of information about the characteristics of masticatory and perioral muscles of the subjects and we can evaluate the effects and results of the applied orthodontic therapy. If we take into consideration the fact that orthodontic skeletal discrepancies with soft tissue dysfunctions are often treated using myofunctional appliances, which in turn have an impact on the surrounding muscles, in the form of neuromuscular adaptation, then electromyography certainly can be applied as a successful, simple and most importantly, objective method for evaluating of the success of orthodontic treatment. 


\section{Aim Of Study}

The purpose of this paper is to compare several different studies, which have been described throughout the literature, which subject of interest was evaluation of masticatory function by determining masticatory efficiency. Also, to determine differences in muscle activity of masseter muscles and perioral muscles in individuals with malocclusions Class II compared with individuals with neutrocclusion. Finally, to evaluate the effects of two different types of myofunctional appliances (active and passive type) on muscle activity, applicable in everyday orthodontic practice in treatment of malocclusions class II, harmful oral habits and soft tissue dysfunction.

\section{Material And Method}

This review includes 10 studies, available through online Internet search based on PubMed. The search was carried out under the key words "mastication" "masticatory efficiency", "Class II malocclusions," "myofunctional therapy", "electromyography", "masticatory muscles." Moreover, taking into consideration our main subject of interest, the attention was directed to studies that applied functional methods, with special emphasis on electromyography, as an objective assessment method of masticatory function and determination of EMG properties of the masticatory and perioral muscle activity in individuals with Class II, and EMG effects in individuals treated with myofunctional orthodontic therapy. In order to examine the function of the masticatory system, specific functional task needs to be performed. For this purpose, a test-food or test-material is used. Different types of test-food were included in different studies, mostly groceries from everyday life, like bread, peanuts, carrots, hazelnuts, walnut, almond, barley, coconut meat, while on the other hand, gelatin, gum, beans soy or silicone rubber were used as a test-material.

According to its consistency, test-food or test-material can be: hard, medium hard or soft. Both, consistency and volume of the test-food or test-material have a major impact on mastication act. Therefore it is necessary that they are standardized for each respondent.

\section{Results}

\subsection{The influence of malocclusion on masticatory performance}

At the begining of the new century, in 2002, Buschang, Throckmorton и English [1] perforemed a study "Does malocclusion affects masticatory performance?" with main purpose to evaluate the largely untested assumption that malocclusion negatively affects masticatory performance. The study included a sample of 185 untreated subjects (48\% male and 52\% female) from 7 to 37 years of age, representing subjects with normal occlusion (n 5 38), Class I (n 5 56), Class II (n 5 45), and Class III (n 5 46) malocclusion. Masticatory performance was evaluated objectively using artificial (CutterSilt, median particle size and broadness of the distribution) and real foods (number of chews for jerky and almonds), and subjectively using a visual analog scale. Their results showed that Class III subjects reported the greatest difficultly, followed by Class II subjects, Class I subjects, and subjects with normal occlusion, respectively. It was concluded that"malocclusion negatively affects subjects' ability to process and break down foods".

In 2010, Periera, Magalhaes, Marques, Gameiro[2]performed “A systematic review: The influence of malocclusion on masticatory performance." A literature survey was done by applying the Medline database in the period from January 1965 to June 2009, using the "Medical Subject Headings". The articles were separated into two main topics: (1) the influence of malocclusion treatment (orthognathic surgery) and (2) the influence of malocclusion type and severity. The search strategy used identified 78 articles. After selection according to the inclusion/exclusion criteria, 12 articles qualified for the final analysis. The research quality and methodological soundness were high in one study, medium in 10 studies, and low in one study. "Malocclusions cause decreased masticatory performance, especially as it relates to reduced occlusal contacts area. The influence of malocclusion treatment (orthognathic surgery) on masticatory performance is only measurable 5 years after treatment".

\subsection{EMG activity in subjects with Class II}

Back in 1973 Ahlgren, Ingervall and Thilander [3], examined the "Muscle activity in normal and abnormal occlusion."The muscle activity was compared in the mandible resting state, during mastication and swallowing in 15 boys with normal occlusion and 15 boys with Class II/1. EMG activity was recorded unilaterally on the anterior and posterior $\mathrm{m}$. temporalis, $\mathrm{m}$. from masseter and superior $\mathrm{m}$. orbicularis oris. No difference was demonstrated in EMG activity of the muscles in a state of physiological dormancy. But "boys with abnormal occlusion tended to show less EMG activity during mastication than boys with normal occlusion. In both types of occlusion, masticatory activity was higher in front of the temporal muscle, rather than in the back. Children with malocclusions showed less EMG activity during swallowing in front of the temporal muscle and masseter than those with normocclusion. In subjects with malocclusions, the length of swallowing activity 
was greater in the back parts of the temporal muscle, while in those with normal occlusion, conversely, the activity was longer in front parts of the temporal muscle. "

Several years later, in 1980, quantitative analysis of EMG activity of the masticatory muscles "Activity of the temporal and masseter muscles in class II, division 1 malocclusions. An electromyographic investigation" was performed in homogeneous groups of boys with Class II, division 1 malocclusion and normal occlusion by Pancherz. H. [4]. Integrated EMG recordings from the temporal and masseter muscles were analyzed during maximal biting in the intercuspal position (centric occlusion) and during the chewing of peanuts. The conclusion showed that "high positive correlations were found between the EMG activity during maximal biting and chewing for both muscles in the two occlusion groups. The impaired muscle activity found in the Class II cases may be attributed to a diverging dentofacial morphology and unstable occlusal contact conditions".

Since electromyography has been proved as a useful method for functional evaluation of occlusal dysfunctions, in 1990, Antonini G, Colantonio L, Macretti N, Lenzi GL. [5] used it in their study "Electromyographic studies in grade II Class 2 and Class III malocclusions." The activity of the masseters and temporal muscle was tested in 6 subjects with malocclusions class II/2 and 7 subjects with Class III malocclusions. The results showed "significant differences in the activity of the masseters during masticatory performance and swallowing between the two groups."

In 2002, the team of Palomari, Vitti, Tosello Dde, Semprini, Rodrigues [6], researched the "Electromyographic study of the masseter muscle in individuals with Class II malocclusion." The purpose of his research was to examine the EMG activity of masseter muscle and analyze the difference of action potential generated by several pre-established jaw movements in individuals with Class II malocclusion. It was studied nine young individuals from both sexes, with ages ranging from 17 to 35 , with no history of orthodontic treatment and/or having some kind of alteration in the temporomandibular joint (T.M.J.)..In order to collect the electromyographic signs a TECA TE-4 Electromyograph and surface electrodes type Beckman were used. The data were submitted to nonparametric statistical analysis by Friedman test. The response variables left occlusive contact, free protraction, protraction with occlusive contact, incisor mastication, right molar mastication and forced central occlusion movements showed significant outcomes. Significant results were shown, and it was concluded that "as the occlusive balance suffers alterations, the malocclusions become predominant and the masseter muscle undergoes functional and structural modifications".

\subsection{EMG activity in subjects with Class II treated with myofunctional appliances}

Aggarwal, Kharbanda, Mathur, Duggal, and Parkash [7] examined "Muscle response to the twin-block appliance: an electromyographic study of the masseter and anterior temporal muscles, " in 1999. The study included 10 girls with Class II/1 and mandibule retrusion treated with twin-block. Changes were registered at the beginning of treatment, after one month of treatment, after 3 months of treatment and after 6 months of treatment, in rest position, during swallowing and at maximal clench. The results showed a significant increase in postural and maximal clenching EMG activity in masseter and a numeric increase in anterior temporalis activity during the 6 month period of treatment. The increased electromyographic activity can be attributed to an enhanced stretch (myotatic) reflex of the elevator muscles, contributing to isometric contractions. The main force for Twin-block treatment appears to be provided through increased active tension in the stretched muscles (motor unit stimulation) and from initiation of myotatic reflex activity and not through passive tension (viscoelastic properties) of jaw muscles. "The results of this study reaffirm the importance of full-time wear for functional appliances to exert their maximum therapeutic effect by way of neuromuscular adaptation."

In 2010, Shubhangi Amit and Toshinval [8] conducted "A clinical, cephalometric, electromyographic and ultrasonographic evaluation of twin block appliance." The response to treatment was evaluated in 20 subjects, aged 10-13 years, for a period of treatment of 6 months. Clinical, cephalometric, electromyographic and ultrasonographic analysis were performed. The EMG results showed lower muscle activity during maximal clench in subjects with class II compared with the control group. The improved results in these subjects after 6 month-treatment with Twin Block, indicate neuromuscular adaptation. Ultrasonographically morphological alteration in the length of masseteric muscle was confirmed. Their conclusion is that "Twin Block appliance is an effective tool that leads to a significant reduction in overjet".

In 2010, Yagci, Uysal, Kara and Okesim [9], using electromyography examined "The effects of myofunctional appliance treatment on the perioral and masticatory muscles in class II, division 1 patients". The tests covered 20 children treated with preorthodontic trainer appliances. Electromyographic examinations were made at the beginning and end of POT therapy during maximal clenching, swallowing, and sucking. The results showed reduced activity of the anterior temporal muscle, masseter muscle and mental muscle at maximal clench. The conclusion of their study confirms that "During the 6 months of POT treatment, the perioral and masticatory muscles of Class II, Division 1 patients improved significantly."

Later, Satigo, Silin, Ramirez [10] in 2014 conducted a study "Electromyographic muscular activity improvement in Class II patients treated with the pre-orthodontic trainer". Their goal was to determine changes 
in the amplitude of the EMG activity of masseteric and temporal muscles at clench in children with Class II division 1 treated with passive myofunctional appliances for a period of 12 months. The study involved 36 children with malocclusions II/1 treated with myofunctional appliances, then 22 children of similar age with malocclusions II/1 that weren't treated and 20 children with normal occlusion as control group. The results showed changes only in the treated group, in which the amplitude values increased at the end of treatment, and according to their values were similar to the control group, unlike the untreated group, in which the values remained low. "Functional trainer appliances increase the activity of masseteric and temporal muscles at clench in subjects with class II/1".

\section{Discussion}

Electromyography is a graphic, neurophysiological and objective method for evaluation of the masticatory function by registering the electrical potentials of the masticatory and perioral muscles. Due to its advantages, such as the objectivity, it has often been the chosen method of many authors. Thanks to the neuromuscular adaptation that is achieved by implementation of the active and passive myofunctional appliances, they present a great tool for improvement of the masticatory function. Numerous studies which main subject of interest was myofunctional treatment in class II, shows its great significance.

\section{Conclusions}

According to the results of this study, electromyographic features of muscle activity of masticatory and perioral muscles confirm that there are differences in masticatory function in subjecs with malocclusion Class I and Class II, as well as between Class II /1 and Class II /2. We can conclude that the implementation of orthodontic myofunctional treatment in subjects with Class II malocclusions have a positive impact on the masticatory function.

\section{References}

[1]. Jeryl D. English, P.H. Buschang, G.S. Throckmorton, Does malocclusion affect masticatory performance?,Angle Orthodontist, 72(1), 2002

[2]. Periera, Magalhaes, Marques, Gameiro, The influence of malocclusion on masticatory performance, Angle Orthodontist, 80, 2010

[3]. Johan G.A. Ahlgren, Bengt F. Ingervall, Birgit L. Thilander, Muscle activity in normal and postnormal occlusion, American Journal of Orthodontics, 64(5),1973, 445-456

[4]. Pancherz H.Activity of the temporal and masseter muscles in class II, division 1 malocclusions.An electromyographic investigation.American Journal of Orthodontics, 77 (6),1980, 679-688

[5]. Antonini G, Colantonio L, Macretti N, Lenzi GL. Electromyographic findings in Class II division 2 and Class III malocclusions.ElectromyogrClinNeurophysiol. 30(1): 1990,27-30.

[6]. Palomari ET, Vitti M, ToselloDde O, Semprini M, Rodrigues AL. Electromyographic study of the masseter muscle in individuals with Class II malocclusion. ElectromyogrClinNeurophysiol. 42(2), 2002, 71-7.

[7]. Aggarwal P, Kharbanda OP, Mathur R, Duggal R, Parkash H., Muscle response to the twin-block appliance: an electromyographic study of the masseter and anterior temporal muscles, Am J OrthodDentofacialOrtop. 116(4):1999, 405-14

[8]. AmitS..Toshniwal N G.,A clinical,cephalometric, electromyographic\&Ultrasonographic evaluation of twin block appliance, J Indian OrthodSoc, 2010; 44(1): 5-16

[9]. Yagci, Uysal, Kara и Okesim, The effects of myofunctional appliance treatment on the perioral and masticatory muscles in class II, division 1 patients, World J Orhod 11:2010; 117-122

[10]. Satigo E. A., Silin A V, Ramirez G. O., Electromyographic muscular activity improvement in Class II patients treated with the preorthodontic trainer, J ClinPediat Dent, 38 (4):2014, 380-384 